\title{
Types of Low Frequency Instabilities in Rotating Detonation Combustors
}

\author{
Vijay Anand and Ephraim Gutmark
}

\begin{abstract}
Rotating detonation combustors (RDC) offer a significant prospective increase in stagnation pressure across it owing to the presence of one or more rotating detonation waves spinning inside the combustor at the kilohertz regime. Naturally, considerable research impetus has been directed towards this technology in recent years to understand the driving mechanics to harness the associated potential of pressure gain combustion (PGC). One such area of focus has been the off-design operating modes of these devices which cause a myriad of instabilities. The current paper is focused towards the discussion of one such instability regime-low frequency instabilities (LFI) - in RDCs. We review three types of LFIs in RDCs based on prior findings, and propose mechanisms for the same.
\end{abstract}

Keywords Detonation - Pressure gain combustion - Combustion instability Low frequency instability $\cdot \mathrm{RDE}$

\section{Introduction}

The supersonic combustion phenomenon of detonation produces a pressure gain of 13-55 in gases [1] across the wave due to the shock wave linked to the combustion front. This detonative mode of combustion is theorized to provide the highly sought after pressure gain (or more specifically, the gain in stagnation pressure, according the AIAA Pressure Gain Combustion Technical Committee) across the component. While pulsed detonation combustors (PDCs) were the widely investigated type of pressure gain combustion (PGC) systems, the majority of recent research has migrated to rotating detonation combustors (RDCs). The higher power density [2], the lack of a need to regulate periodic ignition and fuel/oxidizer injection,

\footnotetext{
V. Anand $(\varangle) \cdot$ E. Gutmark

Department of Aerospace Engineering and Engineering Mechanics, University of Cincinnati, Cincinnati, OH 45221, USA

e-mail: ganeshvn@mail.uc.edu

URL: http://www.uc.edu 
as opposed to a PDC, and the steadier exit flow profile [3] circumvents the notable issues besetting PDCs. Despite the considerable progress made till date on the different facets of RDCs, substantial research is still warranted to ascertain the physics and apply RDCs as a real-world, power-generation device. Until recently, the probable efficiency increases afforded by RDCs, due to detonative burning [4], remained a figment of numerical or analytical solutions, with studies claiming: a notable increased in total impulse over pulsed detonation combustors [3], an increase of up to $9 \%$ in fuel efficiency [5], an increase of up to $15 \%$ in the total pressure in the combustor due to detonation [6], an increase of 5\% in thermal efficiency [7], an increase in thermal efficiency of $1.6 \%$ [8], and finally up to $14 \%$ increase in power plant efficiency over conventional J class turbines [9].

Multiple factors like the reactants type, combustor geometry, thrust, oxidizer and fuel flow rates, and the rotating detonation wave speed at these conditions have been studied. The next obvious step is to identify and understand the various instabilities in RDCs. The field of combustion instabilities in RDCs is extremely nascent in comparison to the monumental work done in addressing the instabilities in gas-turbine combustors and rocket engines. Though the frequency of the instabilities is more often than not a function of the geometry of the combustor, historically speaking, it has been beneficial to group the instabilities this way since the underlying mechanism is generally relatable for a given frequency range. Traditionally, low frequency instabilities having a frequency range of $1-500 \mathrm{~Hz}$ are termed LFI, while those between $500-1000 \mathrm{~Hz}$ are termed intermediate frequency instabilities (IFI, or "buzzing" in rocket engines), and those greater than $1000 \mathrm{~Hz}$ are named high frequency instabilities (HFI) [10]. Our prior publications present the notable parallels between HFI in rocket engines, and rotating detonation and longitudinal pulsed detonations (LPD) in RDC [11, 12]. However, few studies exist at present that have addressed RDC instabilities to any appreciable degree [13-17].The authors have addressed the four prominently occurring instabilities in an RDC of which LFI are a part of, and subsequently speculated on their mechanism in Ref [13]. We use this abbreviation (of LFI) to be in continuation with the terminology used in gas-turbine combustors and rocket engines.

LFI in an RDC seems to be almost ubiquitous. A brief analysis of the pressure-time traces published by the different RDC facilities worldwide gives concrete evidence of the overarching existence of this instability [13, 15, 18-27]. However, most studies have not made an effort to address either the existence, or the mechanism behind LFI. Considering the crippling effects of LFI in rocket engines, supersonic inlets and hypersonic vehicles owing to their tendency to couple with the natural resonant frequency of the structure [28, 29] and the supply feeds [10, 30], it is imperative to acknowledge and treat LFI as we move forward with RDC research. The current study presents a review of the three types of LFI observed in RDCs, from our prior publications $[11,14,22,31]$. It is emphasized here that though the first two types are only observed so far in annular RDCs, whereas the third one has been seen only in a hollow RDC, the driving mechanisms do not limit their occurrence to a specific geometric variant of the combustor, and should be construed to be possible in either combustor geometry at amenable conditions. 


\section{Experimental Methodology}

The air and fuel flow rates through the RDC are controlled by a closed-loop system of nitrogen-driven pilot regulators and a set of Flowmaxx sonic nozzles. The equivalence ratios discussed henceforth are global values estimated from the stagnation pressure and temperature upstream of the choked sonic nozzle. Norgren VP50 proportional control valves (pilot) are linked to Norgren pilot-operated regulators to isolate electrical components from the primary fuel supply. GE Unik 5000 sensors are linked to the choked-flow nozzle assemblies to monitor air and fuel flow rates. Fuel flow is administered to the rig through a pneumatically-actuated Bi-Torq isolation ball valve located just upstream of the fuel plenum, which allows fuel flow rates to stabilize within $2 \mathrm{~s}$ of fuel introduction. The static pressure evolution inside the air plenum, fuel plenum and combustor before and after ignition can be seen in Ref [22]. The systematic error in flow measurements is only from the scaling error of the instruments. The relative errors in the static pressure sensor and thermocouple are known from the associated instrument specifications. This is used to attain the uncertainty in the pressure and thermocouple sensors used in the reactants delivery, and is found to be \pm 0.069 bar and $\pm 1 \mathrm{~K}$ (at the maximum output), respectively, which in turn causes negligible errors in the measured flow rates. For further information about the facility, please consult Refs [31-33].

For the purposes of the current paper, two geometric variations running under air flow rates $\left(\dot{m}_{a}\right)$ of $0.2,0.3$ and $0.4 \mathrm{~kg} / \mathrm{s}$ are to be discussed: annular RDC (running on $\mathrm{H} 2$-air [33]) and hollow RDC (C2H4-air [11]). A side and front-view schematic of the two RDCs and the associated instrumentation is shown in Fig. 1. Air and fuel are supplied from two separate plenums (blue and green, respectively) to attain nonpremixed mixing. Air is injected radially inwards through a circumferential slot and fuel is injected axially through a circumferentially distributed array of fuel orifices. The mixing scheme is generically visualized in Fig. 1. Dimensions of the parts of interest for both the RDCs are presented in Table 1. The reactants mix through this orthogonal slot-orifice injection scheme and are ignited by an initiator tube (see figure). This tube is fed with opposing jets of ethylene and oxygen that are supplied at the headend through Parker solenoid valves. The presence of a detonation wave inside the pre-detonator tube is verified using two ionization probes (not shown here) that accurately capture the speed of the supersonic wave. For a detailed description of the pre-detonator system, we direct the attention of the readers to our previous work [34]. This initial blast wave from the initiator tube is theorized to cause a complex deflagration-to-detonation transition (DDT) mechanism [34] that finally results in the formation of a detonation wave into the RDC channel (red area).

Both the annular and hollow RDC have four rows of instrumentation ports arranged axially in the combustor. This four row instrumentation scheme is present in three sectors of the combustor (see figure) to spatially resolve the rotating detonation wave dynamics as it propagates azimuthally. Note that the color scheme used to represent the three stations in the two RDCs are used later to denote the pressure/ionization traces acquired from the respective sectors. There are also three 


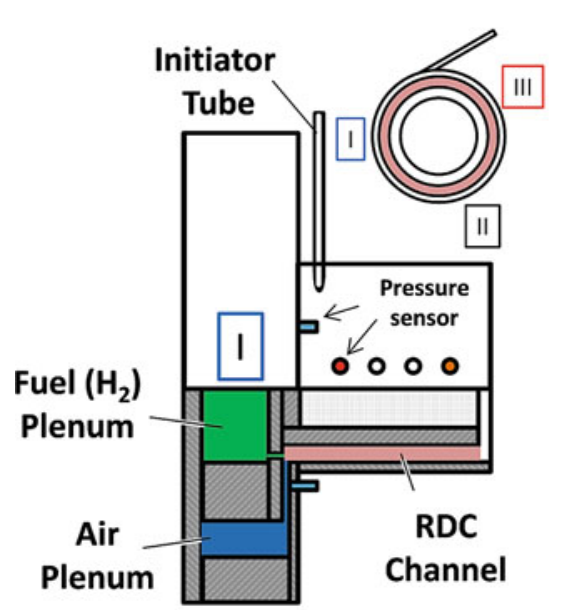

(a) Annular

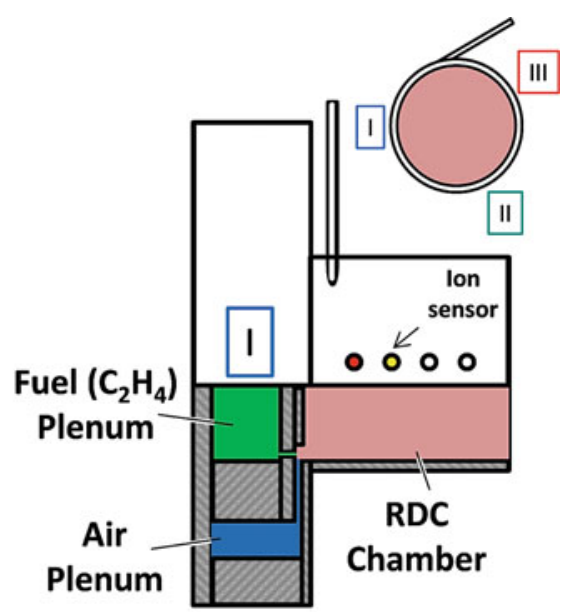

(b) Hollow

Fig. 1 Schematic with instrumentation of a 6" annular RDC, b 6" hollow RDC

Table 1 Dimensions of interest-annular and hollow RDC

\begin{tabular}{|c|c|c|c|}
\hline Part & Geometry measured & $\begin{array}{l}\text { Annular RDC } \\
\text { dimensions }\end{array}$ & $\begin{array}{l}\text { Hollow RDC } \\
\text { dimensions }\end{array}$ \\
\hline Fuel injection & Length/diameter of each hole & 17 & 17 \\
\hline Pattern & Circumferential rows & 3 & 1 \\
\hline \multirow[t]{2}{*}{ Air injection slot } & Slot width & $1.02 \mathrm{~mm}$ & $1.02 \mathrm{~mm}$ \\
\hline & Total slot area & $490 \mathrm{~mm}^{2}$ & $90 \mathrm{~mm}^{2}$ \\
\hline \multirow[t]{5}{*}{ Combustor channel } & Width & $7.5 \mathrm{~mm}$ & - \\
\hline & Inner diameter & $139 \mathrm{~mm}$ & - \\
\hline & Outer diameter & $154 \mathrm{~mm}$ & $154 \mathrm{~mm}$ \\
\hline & Annulus area & $760 \mathrm{~mm}^{2}$ & - \\
\hline & Length & $125 \mathrm{~mm}$ & $125 \mathrm{~mm}$ \\
\hline
\end{tabular}

ports arranged azimuthally in the air inlet (flush-mounted as well) that allow high speed sensing of the air supply plenum dynamics. When there is no sensor in a particular port, a bolt of the proper sizing is used to cover the holes, and is represented as hollow circles in the schematic. In this way, multiple sensor arrangements are possible to analyze RDC dynamics. For the annular RDC, a total of six high speed PCB piezoelectric sensors are used, with three sensors in the combustor in row 1 (red circle in station I in the side view) and three sensors in the air inlet (blue tabs in the side view). Note that this similar arrangement is implemented for the sectors II and III as well. A piezoresistive Kulite sensor in infinite tube pressure (ITP) configuration [35] is integrated to row 4 of the annular RDC, as well. In the hollow RDC, there are three PCB sensors in row 1 (all three sectors) of the combustor. In addition to this, there are also three high speed ion probes (orange circle) in row 2 of the same 
three stations. This allows us to record the coupling between the shock wave and the combustion wave that together form a detonation wave. The first row of instrumentation ports is $1.9 \mathrm{~cm}$ away from the headwall, whereas the other rows are spaced 2.54 $\mathrm{cm}$ from each other. The air inlet ports are $2.5 \mathrm{~cm}$ away from the annulus, radially. Both the RDCs are also instrumented with capillary tube averaged (CTAP) low speed static pressure sensors [35] that monitor the nominal average static pressures before and during RDC hot-fire operation. Data acquisition is at $1 \mathrm{MHz}$ for the high speed sensors and $1 \mathrm{kHz}$ for the low speed ones.

\section{Results and Discussion}

\subsection{Acoustics-Induced LFI: Spatially Homogenous Oscillations}

As mentioned earlier, most operating conditions in an atmospheric annular RDC is prone to a low frequency instability characterized by amplitude modulation in the combustor that is ensconced onto the detonation wave propagation frequency (carrier frequency). This is clearly seen in the piezoresistive pressure trace provided in Fig. 2, which shows the sinusoidal pressure evolution characterized by "waxing and waning" of the peak detonation wave strength. However, two different mechanisms, pertaining to the supply plenum, appear to cause this amplitude modulation (AM) in the combustor. The first mechanism-acoustic oscillation in the form of frequency modulation in the air inlet - is discussed in this section, whereas the next section deals with the other mechanism-detonation wave interaction with the supply plenum. The former manifests as a spatially homogenous LFI, i.e. the sinusoidal oscillation are continuous across all three sectors of the RDC. Remarkably, this type of simultaneous
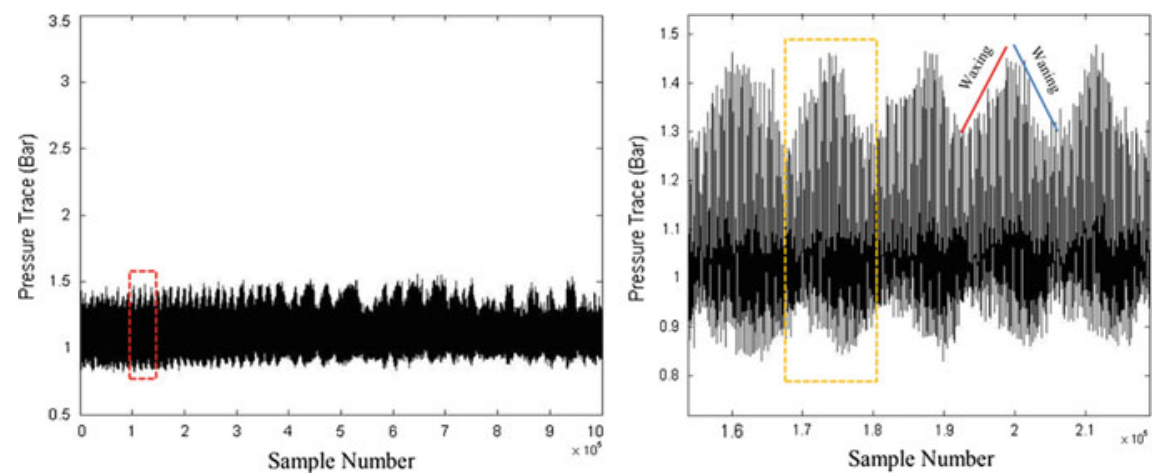

Fig. 2 Pressure-time trace from ITP-mounted piezoresistive sensor at $0.2 \mathrm{~kg} / \mathrm{s}$ at $\Phi=1.8$ (left), and a magnified section from the same test point (right) 
(a)

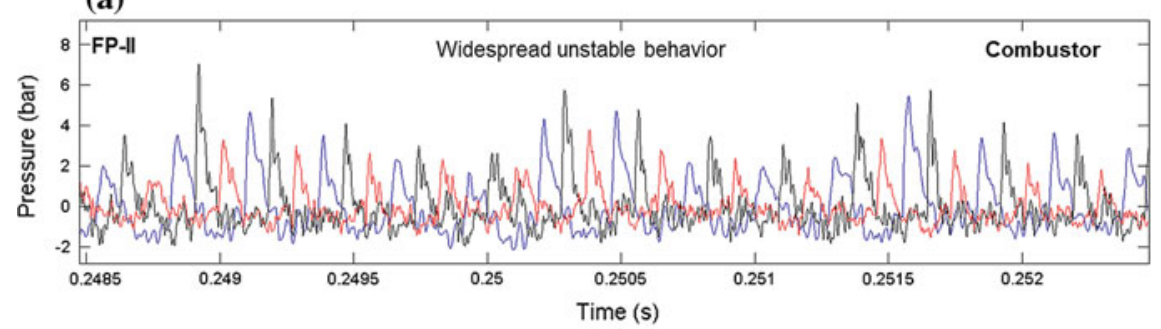

(b)

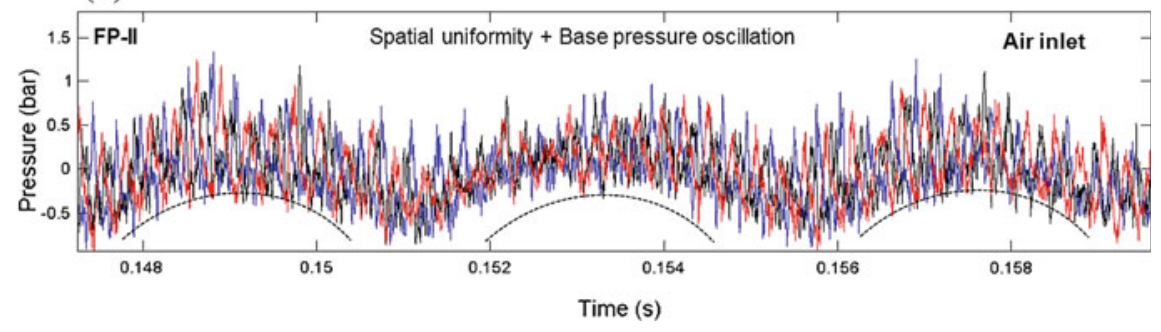

Fig. 3 a Pressure series from the combustor, b pressure series from the air inlet $(0.4 \mathrm{~kg} / \mathrm{s})$ showing FM LFI

oscillation is observed in rocket engines at unstable conditions of operation when there is an onset of high frequency combustion instabilities, and is referred to as "chugging" [36], to refer to the changes in flow rates caused by this type of acoustic oscillation linked to the plenum [10]. The latter results in a spatially non-homogenous LFI that is discontinuous across the different RDC sectors, i.e. this LFI appears to be revolving about the combustor annulus. This type of phase-lagged low frequency rotating instability has been observed in rocket engines, as well (Fig. 9.7.1i in [10]). There, it is called a "precessing tangential mode" and is noted to have anywhere between five to hundred tangential wave laps per low frequency cycle.

Figure $3 \mathrm{a}$ is an arbitrary pressure trace from the combustor that shows unstable detonation wave propagation that varies sinusoidally in strength as the detonation wave moves circumferentially through the three instrumented sectors of the RDC, implying a continual variation in the rotating detonation wave strength. Fig. $3 \mathrm{~b}$ gives a pressure time series in the air inlet that shows an oscillation in the base pressure (tagged by dotted curves). The carrier signal in the air inlet is the leaked shock wave from the detonation propagation through the three sectors. This carrier pressure signal, however, can be seen to have a sustained base pressure modulation (thereby, being frequency modulated) that is azimuthally simultaneous. That is, all three sectors are prone to sinusoidal increases and decreases in the base pressure. We will call this spatial homogeneity.

Since spatially homogenous LFI is linked to the base pressure oscillation (chugging) in the air inlet, it is desirable to analyze the oscillation in the air inlet. Fig. 4a shows the Fast Fourier Transform (FFT) plot for three stoichiometric test cases at different air flow rates. While the ff (the RDC operation frequency which is equal to the 

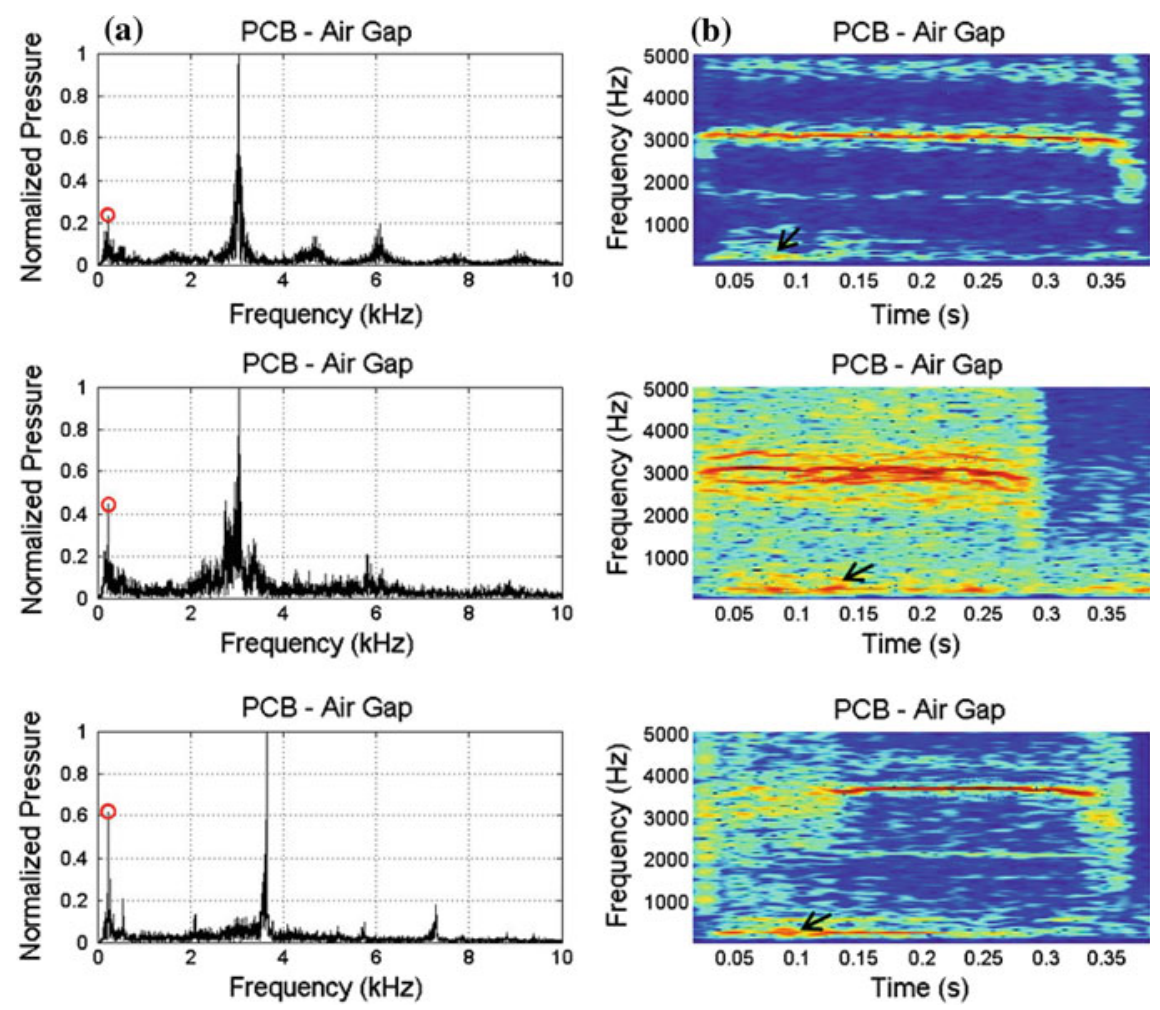

Fig. 4 FFT plot a and Spectrogram $\mathbf{b}$ showing the spatially homogenous LFI frequency in the air inlet for $0.2 \mathrm{~kg} / \mathrm{s}$ (top), $0.3 \mathrm{~kg} / \mathrm{s}$ (middle) and $0.4 \mathrm{~kg} / \mathrm{s}$ (bottom) —all flow rates at an equivalence ratio of 1.0

detonation wave frequency) is easily observable from the three plots at $\mathrm{f} \approx 3 \mathrm{kHz}, \mathrm{f} \approx$ $3 \mathrm{kHz}$ and $\mathrm{f} \approx 3.8 \mathrm{kHz}$ respectively, there is also considerable LFI activity in all three test cases at $\mathrm{f} \leq 0.5 \mathrm{kHz}$ (the frequencies surrounding the red circle in the figure). The secondary dominant frequency is identified to be $\mathrm{f} \approx 235 \mathrm{~Hz}$ (shown by red circle) and is the same across $0.2,0.3$ and $0.4 \mathrm{~kg} / \mathrm{s}$ across three different equivalence ratios at the three air flow rates [22]. This is an interesting discovery since it implies that irrespective of the air flow rate (or in other words, the air plenum pressure) or the equivalence ratio of the operating point, the inlet of the RDC in use for the current study always oscillates at $\mathrm{f} \approx 235 \mathrm{~Hz}$. The $\mathrm{f} \approx 235 \mathrm{~Hz}$ oscillation (black arrow) does not extend throughout the test, but is rather concentrated for a few seconds after ignition, as shown by the spectrogram, in Fig. $4 \mathrm{~b}$ respectively. To confirm that the oscillation is induced by the detonation wave and not due to an inherent oscillation in the air supply, the RDC was operated under cold-flow conditions without ignition of the reactants, which revealed the lack of any activity in the $235 \mathrm{~Hz}$ region. Since the cold flow RDC testing did not produce any oscillation at the same frequency, it can be understood that the oscillation in the air gap is linked to detonation wave propagation in the annulus. The Helmholtz frequency for the air plenum under study 
is estimated to be $\approx 355 \mathrm{~Hz}$ for all flow rates since the speed of sound is relatively unaltered due to an almost constant supply temperature. Here, the length of the radially oriented slot (see Fig. 1) is taken to be the length of the resonator neck, with an added end correction of 0.6 times the equivalent diameter (obtained from the total air injection area). The 0.6 coefficient is recommended for geometries with two open ends, as is the case with the air plenum. But, the complex geometry of the plenum would skew the resonance frequency obtained from the basic Helmholtz equation considerably [37]. For instance, $\mathrm{a} \approx 15 \%$ error is incurred between the calculated and the experimentally obtained frequency when the basic Helmholtz equation is used to calculate the resonance frequency for a cylindrical prism with a long neck [37]. Hence, to get an accurate resonance frequency value, geometry-specific equations need to be developed, even for simple geometries. Despite these unknown variables, since the approximately estimated resonance frequency is different from the oscillation frequency in the inlet by only $33 \%$, it is a strong indication of the air plenum's functioning as a Helmholtz resonator due to the excitation produced by the high-frequency detonation wave in the combustor. This is not unlike the chugging phenomenon observed in the F-1 engines due to the quarter-wave mode of acoustic excitation produced in the engine's reactants supply lines [10]. It is hypothesized that the detonation wave excites the air inlet to a resonance frequency which manifests as the spatially homogenous LFI in the combustor, due to the fluctuating air supply, which in turn appears to cause a similar fluctuation in the detonation wave's strength.

\subsection{Detonation-Induced LFI: Spatially Non-homogenous Oscillations}

Annular RDC supply plenums (studied here) also interact with the combustor dynamics in a spatially non-homogenous way. Here, spatial non-homogeneity' is defined as the phenomenon where for a given arbitrary temporal window, a particular sector of the RDC exhibits notably higher peak pressures (of subsequent detonation laps) than the other sectors. In this case, while there is still a sinusoidal low frequency oscillation, it is segregated to a given sector of the combustor and air inlet; or in other words the low frequency amplitude modulation revolves about the device. The alternating sectoral strength is depicted in Fig. 5, which shows stronger subsequent waves in the air inlet sector I (blue), followed by sector III (red), and finally sector II (black). This is an amplitude modulation (AM) of the carrier shock wave pressure signal. The facet to observe here is the direction of rotation of the detonation wave (trackable through the leaked shock wave into the air inlet). The rotating detonation wave moves in the counter-clockwise direction (black $\rightarrow$ red $\rightarrow$ blue/sector II $\rightarrow$ sector III $\rightarrow$ sector I) in the represented pressure traces. The spatially varying AM oscillation, however, exhibits the reverse order (blue $\rightarrow$ red $\rightarrow$ black / station $\mathrm{II} \rightarrow$ station III $\rightarrow$ station I). To clarify, the sinusoidal amplitude modulated variation is exhibiting a clockwise rotating motion. This kind of behavior- the spatially varying sinusoidal AM instability 


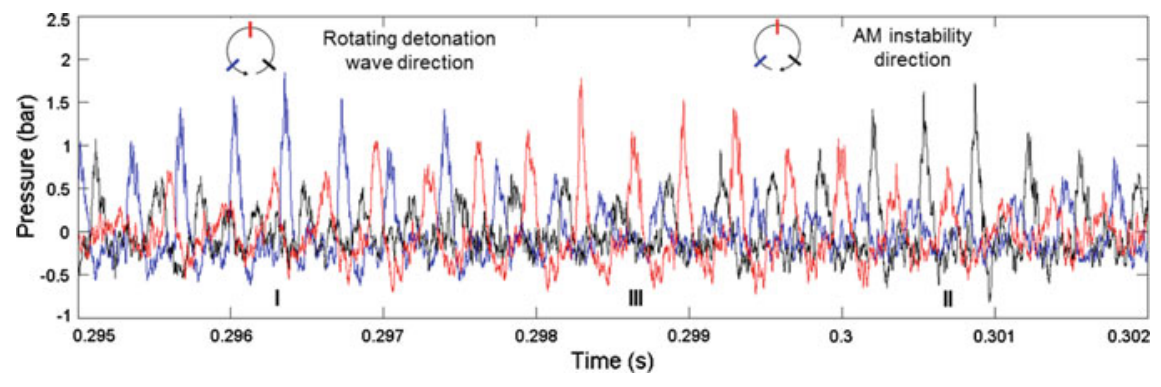

Fig. 5 AM LFI pressure series from air inlet with FP-I at $\dot{m}_{a}=0.2 \mathrm{~kg} / \mathrm{s}, \Phi=1.0$

in the air inlet moving in the opposite direction to the rotating detonation wave- is witnessed when there is spatially non-homogenous LFI. It is thus apparent that AM LFI is characterized by the spatially varying detonation strength is not caused due to a simultaneous stationary acoustic phenomenon in the air inlet unlike the previous case, but rather, a low-speed rotatory event in the air inlet and combustor.

Details of the origins of this rotating instability emerge when an operating point exhibiting the same is studied in entirety. Fig. 6 shows the pressure traces from the three sectors in the air inlet at $\dot{m}_{a}=0.2 \mathrm{~kg} / \mathrm{s}$ and $\Phi=1.0$. From Fig. 6a it can be seen that the rotating detonation wave is spinning counterclockwise and AM LFI (the sinusoidal overarching component) is clockwise. Fig. 6b shows the highly dynamic nature of this instability. Initially, there is incoherence and there is no marked sinusoidal spatially varying oscillation. However, from $t=0.14 \mathrm{~s}$, one can observe easily delineated sinusoidal variation existing until the end of the RDC hot-fire run. Additionally, it could also be seen that the sinusoidal variation gradually "thins" with time, occurring faster as time progresses. This kind of dynamic variation in the frequency of occurrence of a phenomenon is usually termed "bootstrapping", a non-linear phenomenon that has been seen to occur in rocket engines [38], albeit for different reasons. The normalized pressure envelope of the low frequency spatially instability is shown in Fig. 6c for all three sensors. The spatial variation and the faster occurrence of the instability throughout the test can be easily noticed. In fact, the plot of the AM LFI velocity vs. time (Fig. 6d) shows that initially the induced velocity is around $40 \mathrm{~m} / \mathrm{s}$, and continuously increases from $\mathrm{t} \approx 0.135 \mathrm{~s}$ to $\mathrm{t} \approx 0.275 \mathrm{~s}$, after which time it plateaus to a speed of $100 \mathrm{~m} / \mathrm{s}$. The gradual increase in AM LFI velocity until a terminal condition can be better understood by analyzing the detonation wave speed and directionality plot shown in Fig. 6e. The rotating detonation wave establishes in the clockwise direction initially and rotates in this direction until $\mathrm{t} \approx$ $0.135 \mathrm{~s}$. However, at $\mathrm{t} \approx 0.135 \mathrm{~s}$ there is a sudden stochastic flip in the direction to counterclockwise rotation, and from $\mathrm{t} \approx 0.135 \mathrm{~s}$ till $\mathrm{t} \approx 0.34 \mathrm{~s}$, the rotating detonation wave exhibits stable directionality in the counterclockwise direction. It is thus apparent that the steady increase in AM LFI to a terminal value from $\mathrm{t} \approx 0.135 \mathrm{~s}$ is closely linked to the rotating detonation direction, i.e. as long as the detonation wave maintains its direction of rotation, the AM LFI in the air plenum maintains its direction. The amplitude modulation in the air inlet seems to exhibit a considerable 
transient operation followed by an apparent steady-state period where the rotary velocity of AM LFI is plateaued. This terminal rotary speed is approximately 100 $\mathrm{m} / \mathrm{s}$, for the present test condition. This plateau in the rotary speed is not observed when the detonation wave flips direction frequently [31].

From the above observations, we have enough information to propose a mechanism behind the amplitude modulated spatially non-homogenous instability in an RDC. Fotia et al. [39], in their two-dimensional experimental RDC study, have noted that the trailing shock wave (attached to the bottom of the detonation wave, as established by Schwer and Kailasanath [40]) moves at about $60 \%$ of the detonation wave speed. Owing to this relative velocity between the detonation wave in the combustor and the trailing shock wave in the reactants plenum, they postulated a "pressure beating" event when the next detonation wave lap interacts with the trailing shock wave in the plenum from the prior lap. However, their study was a one detonation wave event, and hence the "pressure beating", or more accurately, constructive/destructive interference was not observed. On the other hand, Schwer and Kailasanth [40], in their numerical simulation, have noted that this trailing shock wave (incident wave) travels to the base of the reactants plenum and gets reflected as another relatively strong wave. They tested two different reactant plenum depths (to vary the strength of the reflected wave) and concluded that this reflected wave did not impact the reactants conditions upstream of the next lap of the detonation wave. Additionally, multiple reflected waves are produced at discrete times and locations along the concave surface, depending on curvature and other effects [41]. Evidence for this in an RDC can be seen in the Schlieren images of an air plenum (with curved base) exposed to a detonation wave, obtained by Bedick et al. [42]. Hence, one should expect a similar phenomenon of production of multiple reflected waves moving in the opposite direction of the trailing shock wave when it is incident on the concave curved surface of the base of the air plenum of the RDC. We hypothesize here that the current LFI in an RDC is probably due to this interaction of incident and reflected waves. This is an extension of the acoustic interference hypothesis that Fotia et al. [39] that is expanded to include the effects of the reflected wave as well, in an actual RDC. Naturally, one should expect this complex system of waves to develop much slower in comparison to the detonation wave. This could explain the lower speed of the rotation and the stochasticity in AM LFI when the detonation wave randomly switches direction. In light of this theory, one could contend that the speed of this rotary amplitude modulated motion is inherently a function of the detonation wave speed and the fluidic impedance of the injector element. Increase of the former and decrease of the latter is expected to produce faster moving wave packets. Visualization of the reactants plenum is required to further test the proposed hypothesis.

\subsection{Combustion-Induced LFI: Spatially Homogenous}

As opposed to the previous two cases where LFI is caused in the combustor due to interaction with the supply plenum, in the current case LFI is dictated seemingly by the combustion dynamics alone. Shown in Fig. 7a are the pressure traces (from the 

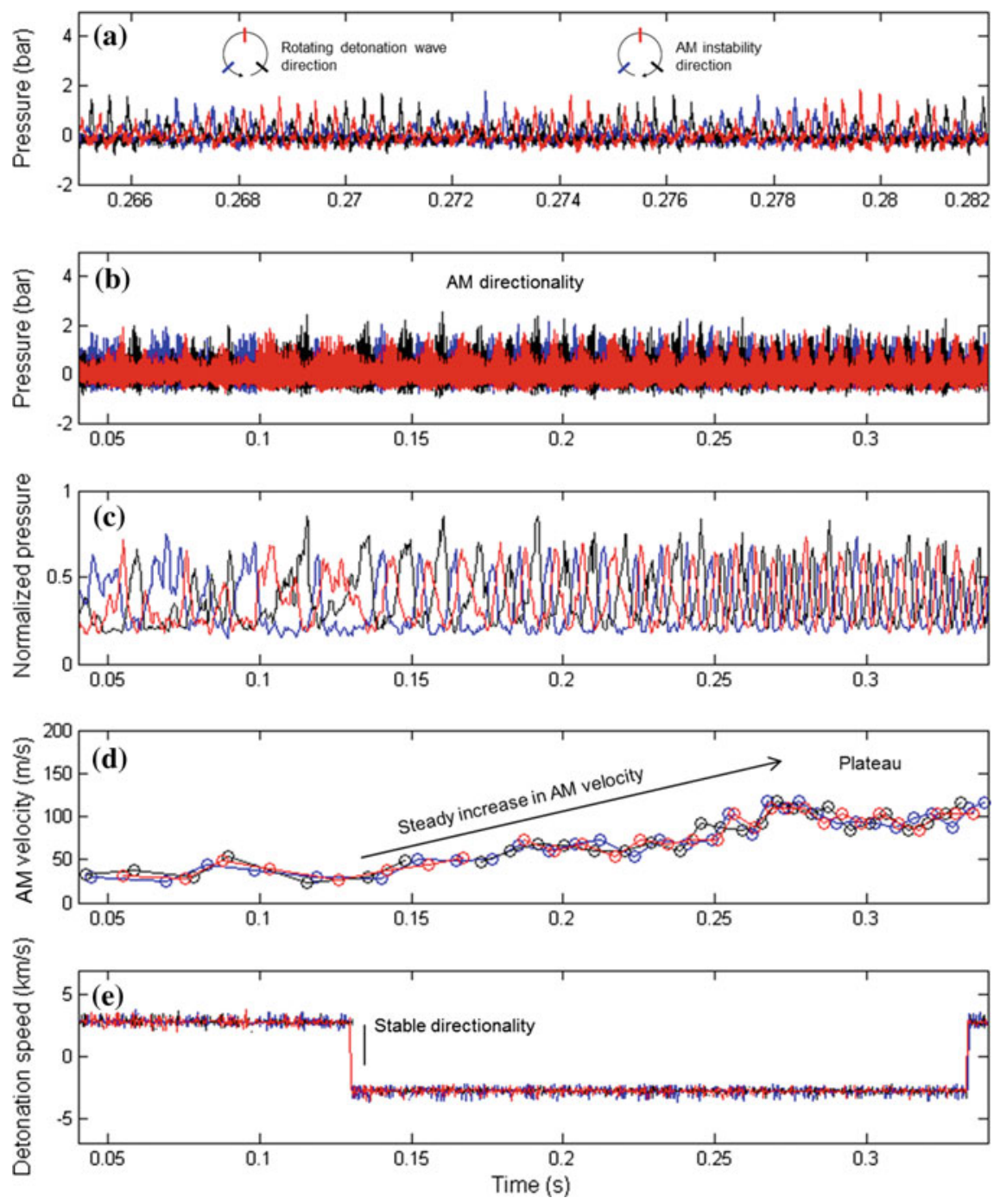

Fig. 6 Variables of interest acquired from air inlet sensors at $\dot{m}_{a}=0.2 \mathrm{~kg} / \mathrm{s}, \Phi=1$

pressure sensor in row 1) and the ionization traces (from the ion sensor in row 2), for one such test point in a hollow RDC, running on ethylene-air mixtures. Note that both sensors are at the same sector/azimuth, which gives us the ability to track the relative changes between pressure and ionization. Upon ignition $(\mathrm{t} \approx 0 \mathrm{~s})$, it is seen from Fig. 7a, that initially there is no pressure activity; just ionization activity. At $\mathrm{t}$ $\approx 0.02 \mathrm{~s}$, rotating pressure waves with considerable peak pressure magnitudes (and hence rotating detonations) appear. However, at $\mathrm{t} \approx 0.05 \mathrm{~s}$, the rotating detonation wave seemingly descends into highly unstable propagations that are characterized 

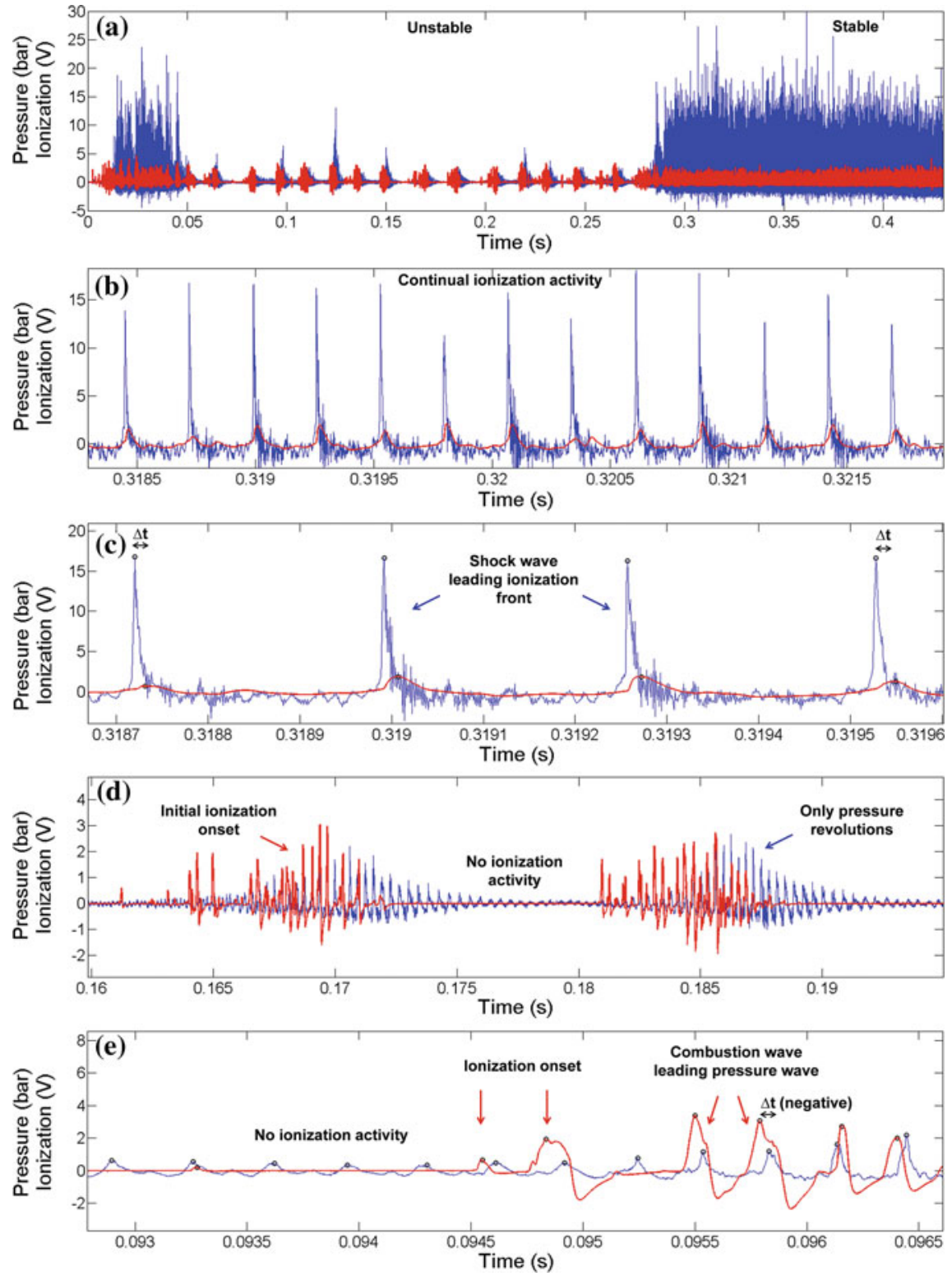

Fig. 7 Pressure and ionization traces at $0.4 \mathrm{~kg} / \mathrm{s}$ and $\Phi=1.6$ : a complete traces, b stable propagation, $\mathbf{c}$ magnified traces during stable propagation, $\mathbf{d}$ unstable propagation, and $\mathbf{e}$ magnified traces during unstable propagation 
by "packets" of subsequent laps that have the AM sinusoidal component. This AM fluctuation is characteristic of all the unstable rotating pressure wave propagations observed in hollow RDCs [11]. This unstable behavior extends till $\mathrm{t} \approx 0.28 \mathrm{~s}$, after which there is, once again, sustained periodic rotating detonations without the packets of instability. Of interest is the fact that, for a given packet of instability, ionization (red) is recorded for only about roughly the first half of the sinusoidal packet, whereas the second half is composed of just pressure activity (Fig. 7a). Figure 7b shows pressure and ionization data from an arbitrary duration from the same test point when there is stable rotating detonation wave propagation. It can be seen that the shock wave precedes the ionization (combustion) peak, as is to be expected in a detonation wave. A magnified image of this snippet (Fig. 7c) shows this distinctionpressure peak preceding the ionization peak-better. The black circles denote the peak pressure and ionization values, and are acquired by a time-of-flight algorithm that captures peak values, for a given lap. Fig. 7d contains pressure and ionization data during two subsequent packets of unstable operation, from the same test case. For a given amplitude modulated packet of subsequent rotating pressure waves, ionization activity exists for roughly the first half of the packets. Periods between the two packets do not exhibit any ionization, but do exhibit very weak rotating pressure waves that do not exceed 0.5 bar. It is once again emphasized here that even during this very low amplitude unstable, AM behavior, the pressure waves are confirmed to be rotating (since we have three circumferentially distributed sensors in row 1). A striking difference from the pressure-ionization coupling behavior seen during stable operation (Fig. 7c) is evident in Fig. 7e, during unstable behavior. During unstable propagation, most laps in a given cycle have the peak pressure succeeding the ionization/combustion peak. This is, in fact, observed for all unstable propagations. Hence, it is imperative to quantify the time lag, $\Delta \mathrm{t}$, between the peak pressure event and the peak ionization event to ascertain the difference between the two propagation mechanisms. We use the system of positive $\Delta \mathrm{t}$ if the peak pressure precedes the peak ionization and negative $\Delta \mathrm{t}$ if it succeeds it.

Figure 8 gives the pressure trace ( 3 sensors in row 1 ), ionization trace ( 3 sensors in row 2), wave speed (from a time-of-flight algorithm) and time lag between pressure peak and ionization peak (negative value if ionization peak precedes pressure peak) for two different test points that exhibit unstable propagation. Note that all plots presented are for the whole duration of testing. The following observations can be readily made. During unstable operation, there are packets of rotating pressure waves with very low peak pressure magnitudes. The corresponding ionization traces reveal a similar packet-type behavior (Fig. 8). The sinusoidal oscillation evident in the peak pressure magnitudes of subsequent laps also results in sinusoidal oscillations in the lap-to-lap wave speeds, when the propagation is unstable. This is suggestive of a periodically strengthening and weakening rotating wave. It is imperative to note that during stable propagation the wave speed of each lap is about $95 \%$ of the C-J speed (red dotted line), whereas during unstable propagation, the wave speed is highest at the highest pressure point of the packet and is about $90 \%$ of the C-J speed. The average wave speed, however, is considerably lower due to the waxing and waning on either sides of the maximum pressure value. Of heightened importance are the 

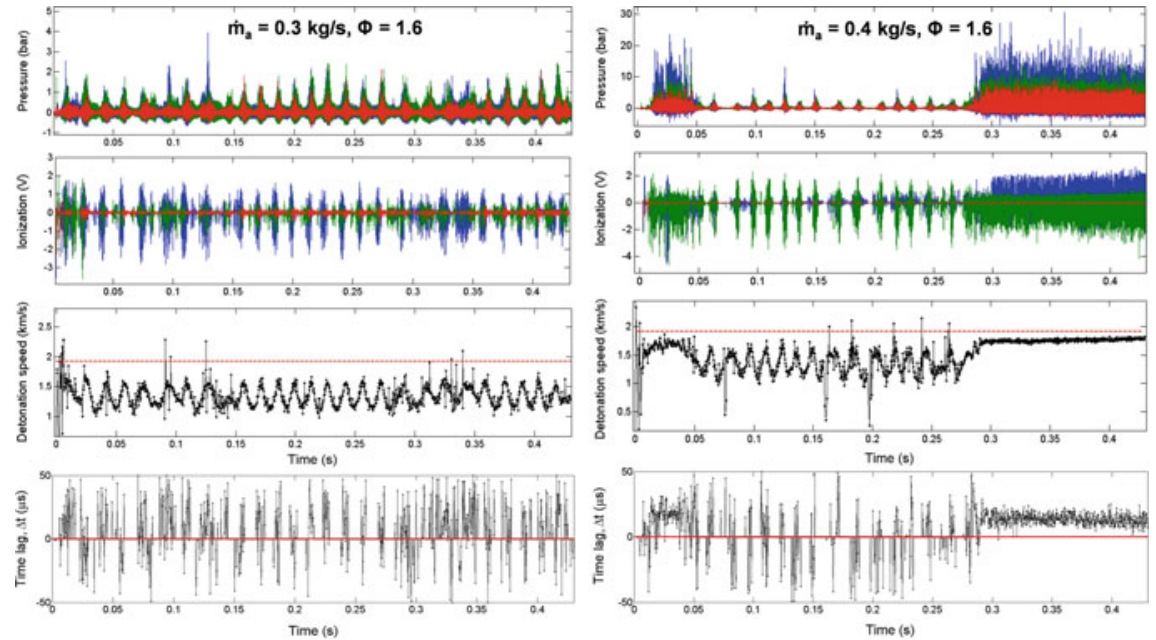

Fig. 8 Pressure traces (first row), Ionization traces (second row), wave speed (third row) and time lag between pressure and ionization wave (fourth row)

time lag characteristics across the different test cases. It is seen that when the propagation is stable, the time lag is predominantly positive across the approximately thousand laps, i.e. peak pressure precedes ionization. Initially, after ignition, combustion wave precedes the pressure wave (region indicated by blue arrow). However, after this initial period, the shock wave supersedes ionization events, thereby exhibiting the commonly known behavior of stable detonation wave propagation. When the propagation is unstable, such an observation is not seen. In fact, during unstable propagation, for a given packet of instability, the number of laps with ionization preceding the pressure wave is almost equal to the number of laps with the opposing trend. A maximum of $\pm 50 \mu \mathrm{s}$ is set to remove fallacious values that are an artifact of the algorithm.

While this result is seemingly contradictory at first glance, it can be readily explained to be due to the flame acceleration and transition to detonation phenomenon, most commonly observed in ducts with deflagration-to-detonation inducing obstacles [43]. In their comprehensive review of the said phenomenon, Ciccarelli and Dorofeev state that "flame propagation in an enclosure generates acoustic waves that, after reflections from walls and obstacles, can interact with the flame front and develop flame perturbations through a variety of instability mechanisms" and say that if such a flame propagation is fast enough, it "can result in severe flame distortion which can induce flame acceleration and, in extreme cases, cause transition to detonation" [43]. One could thus postulate that the concave surface of the RDC outer wall acts like a reflecting obstacle thereby sustaining the propagation process. It is a well-known property of detonations in curved channels to have pronounced collision and subsequently stronger ignitions at the outer concave wall [44, 45]. Thus, finally we can attribute the low magnitude rotating pressure waves to be a type of unstable detonation wave propagation, where ignition seems to be continual and caused due to 
a probable flame acceleration mechanism sustained by the concave wall. Of course, such a claim is a preliminary theory and needs to be validated through focused, future studies. At present, however, it seems reasonable to attribute two different propagation mechanisms - conventional detonation propagation / flame acceleration causing DDT-to stable / unstable AM propagation. An analogous behavior to this would be the change from steady propagation to galloping and eventually stuttering detonations, in planar detonations in tubes, which exhibits a similar type of low frequency oscillation in pressure and wave speed [46].

\section{Conclusion}

The current chapter dealt with the identification and classification of three types of low frequency instabilities in rotating detonation combustors. While all three types are characterized by a sinusoidal amplitude modulation of the rotating detonation wave's peak pressure, their driving mechanisms appear to be divergent. The first two types of LFI are linked to the supply plenum dynamics. For the first case, Helmholtz resonance appears to the driving factor that causes acoustic oscillation in the air inlet which in turn produces spatially homogeneous low frequency instability in the combustor. This type of LFI is similar to the chugging instability widely reported in rocket engines, which is also predicated to depend on supply line and plenum acoustics. The second type of LFI is spatially non-homogenous and is characterized by a slow-moving rotary event in the combustor and the air inlet. This type of LFI is also alluded to in rocket engine instabilities' literature and is termed a precessing tangential mode. We show evidence that this rotary sinusoidal oscillation in detonation peak pressure moves in a direction opposite to the direction of the rotating detonation wave, and subsequently hypothesized it to be caused due to a complex constructive and destructive interference of the shock waves leaked into the supply plenum. This needs to be verified in future studies. Finally, the last LFI type is linked to the combustion wave dynamics itself and is spatially homogenous. It is shown that such LFI is distinguished by a complex interplay between the pressure wave and the combustion wave that together make a revolving front. During stable operation in a hollow RDC, the shock wave precedes the combustion front, whereas during LFI-defined unstable operation in the same RDC, the combustion wave precedes the pressure wave implying the presence of the phenomenon of flame-acceleration in ducts causing a detonation wave, rather than a detonation event itself. This process is similar to the alternative coupling and decoupling of the shock wave and combustion front, which is observed widely in unstable detonation propagation at near-limits in tubes. As noted in the introduction, the field of instabilities analysis in RDCs is nascent in comparison to the decades of research on the same in rocket engines, where there are considerable unknowns even at present. Moving forward, it is imperative to apply a similar impetus in understanding the off-design functioning of RDCs to eventually enable its implementation in real-world applications. 


\section{References}

1. Turns, S.: An Introduction to Combustion. McGraw-Hill International, Singapore (2006)

2. Wolański, P.: Detonative propulsion. Proc. Combust. Inst. 34, 125-158 (2013)

3. Yi, T.-H., Lou, J., Turangan, C., Choi, J.-Y., Wolanski, P.: Propulsive performance of a continuously rotating detonation engine. J. Propuls. Power. 27, 171-181 (2011)

4. Wintenberger, E., Sheperd, J.E.: Thermodynamic cycle analysis for propagating detonations. J. Propuls. Power. 22, 694-697 (2006)

5. Jones, S., Paxson, D.: Potential benefits to commercial propulsion systems from pressure gain combustion. In: Joint Propulsion Conference, San Jose, CA (2013)

6. Frolov, Dubrovskii, A.V., Ivanov, V.S.: Three-dimensional numerical simulation of operation process in rotating detonation engine. Prog. Propuls. Phys. 4, 467-488 (2013)

7. Sousa, J., Paniagua, G., Collado Morata, E.: Thermodynamic analysis of a gas turbine engine with a rotating detonation combustor. Appl. Energy. 195, 247-256 (2017)

8. Strakey, P., Ferguson, D., Sisler, A., Nix, A.: Computationally quantifying loss mechanisms in a rotating detonation engine. In: 54th AIAA Aerospace Sciences Meeting, pp. 1-14 (2016)

9. Sonwane, C., Clafli, S.: Recent advances in power cycles using rotating detonation engines with subcritical and supercritical CO2. In: The 4th International Symposium-Supercritical CO2 Power Cycles, Pittsburgh, Pennsylvania (2014)

10. Harrje, D.T.: Liquid propellant rocket combustion instability, Washington D.C. (1972)

11. Anand, V., St. George, A., Farbos de Luzan, C., Gutmark, E.: Rotating detonation wave mechanics through ethylene-air mixtures in hollow combustors, and implications to high frequency combustion instabilities. Exp. Therm. Fluid Sci. 92 (2018)

12. Anand, V., St. George, A., Driscoll, R., Gutmark, E.: Longitudinal pulsed detonation instability in a rotating detonation combustor. Exp. Therm. Fluid Sci. 77, 212-225 (2016)

13. Anand, V., St. George, A., Driscoll, R., Gutmark, E.: Characterization of instabilities in a rotating detonation combustor. Int. J. Hydrogen Energy. 40, 16649-16659 (2015)

14. Anand, V., George, A.S., Driscoll, R., Randall, S., Gutmark, E.J.: Statistical treatment of wave instability in rotating detonation combustors. In: 53rd AIAA Aerospace Sciences Meeting (2015)

15. Liu, Y., Wang, Y., Li, Y., Li, Y., Wang, J.: Spectral analysis and self-adjusting mechanism for oscillation phenomenon in hydrogen-oxygen continuously rotating detonation engine. Chinese J. Aeronaut. 28, 669-675 (2015)

16. Li, Y., Wang, Y., Wang, J., Li, Y.: Detonation instability of continuously rotating detonation engines for H 2 -Air mixture 1, 1-7 (2014)

17. Frolov, S.M., Aksenov, V.S., Ivanov, V.S., Shamshin, I.O.: Large-scale hydrogen-air continuous detonation combustor. Int. J. Hydrogen Energy. 40, 1616-1623 (2015)

18. Suchocki, J., Yu, S.-T., Hoke, J., Naples, A., Schauer, F., Russo, R.: Rotating detonation engine operation. In: 50th AIAA Aerospace Sciences Meeting including the New Horizons Forum and Aerospace Exposition. American Institute of Aeronautics and Astronautics, Reston, Virigina (2012)

19. Dyer, R., Naples, A., Kaemming, T., Hoke, J., Schauer, F.: Parametric testing of a unique rotating detonation engine design. In: 50th AIAA Aerospace Sciences Meeting including the New Horizons Forum and Aerospace Exposition, Nashville, Tennessee (2012)

20. Shank, J.C., King, P.I., Karnesky, J., Schauer, F.R., Hoke, J.L.: Development and testing of a modular rotating detonation engine. In: 50th AIAA Aerospace Sciences Meeting, Nashville, TN (2012)

21. Kindracki, J.: Experimental research on rotating detonation in liquid fuel-gaseous air mixtures. Aerosp. Sci. Technol. 43, 445-453 (2015)

22. Anand, V., George, A., Driscoll, R., Gutmark, E.: Analysis of air inlet and fuel plenum behavior in a rotating detonation combustor. Exp. Therm. Fluid Sci. 70, 408-416 (2016)

23. Yang, C., Wu, X., Ma, H., Peng, L., Gao, J.: Experimental research on initiation characteristics of a rotating detonation engine. Exp. Therm. Fluid Sci. 71, 154-163 (2016) 
24. Wang, C., Liu, W., Liu, S., Jiang, L., Lin, Z.: Experimental verification of air-breathing continuous rotating detonation fueled by hydrogen. Int. J. Hydrogen Energy. 40, 9530-9538 (2015)

25. Lin, W., Zhou, J., Liu, S., Lin, Z., Zhuang, F.: Experimental study on propagation mode of H2/Air continuously rotating detonation wave. Int. J. Hydrogen Energy. 40, 1980-1993 (2015)

26. Kindracki, J., Kobiera, A., Wolański, P., Gut, Z., Folusiak, M., Swiderski, K.: Experimental and numerical study of the rotating detonation engine in hydrogen-air mixtures. Prog. Propuls. Phys. 2, 555-582 (2012)

27. Peng, L., Wang, D., Wu, X., Ma, H., Yang, C.: Ignition experiment with automotive spark on rotating detonation engine. Int. J. Hydrogen Energy. 40, 8465-8474 (2015)

28. Clayton, R., Rogero, R., Sotter, J.: An experimental description of destructive liquid rocket resonant combustion. AIAA J. 6, 1252-1259 (1968)

29. Narayanaswamy, V., Raja, L.L., Clemens, N.T.: Control of unsteadiness of a shock wave/turbulent boundary layer interaction by using a pulsed-plasma-jet actuator. Phys. Fluids. 24 (2012)

30. Boshoff-Mostert, L., Viljoen, H.J.: Analysis of combustion-driven acoustics. Chem. Eng. Sci. 53, 1679-1687 (1998)

31. Anand, V., St. George, A.: Amplitude modulated instability in reactants plenum of a rotating detonation combustor. Int. J. Hydrogen Energy. 42, 12629-12644 (2017)

32. St. George, A.C., Driscoll, R.B., Anand, V., Munday, D.E., Gutmark, E.J.: Development of a rotating detonation engine facility at the university of cincinnati. In: 53rd AIAA Aerospace Sciences Meeting. American Institute of Aeronautics and Astronautics, Reston, Virginia (2015)

33. Anand, V., St. George, A., Driscoll, R., Gutmark, E.: Investigation of rotating detonation combustor operation with H 2 -Air mixtures. Int. J. Hydrogen Energy. 41, 1281-1292 (2016)

34. St. George, A., Randall, S., Anand, V., Driscoll, R., Gutmark, E.: Characterization of initiator dynamics in a rotating detonation combustor. Exp. Therm. Fluid Sci. 72, 171-181 (2016)

35. Stevens, C.A., Fotia, M.L., Hoke, J.L., Schauer, F.R.: Comparison of transient response of pressure measurement techniques with application to detonation waves. AIAA Aerosp. Sci. Meet. 5, 1102-2015 (2015)

36. Hulka, J.R., Jones, G.W.: Performance and stability analyses of rocket thrust. In: 46th AIAA/ASME/SAE/ASEE Joint Propulsion Conference, Nashville, TN (2010)

37. Alster, M.: Improved calculation of resonant frequencies of Helmholtz resonators. J. Sound Vib. 24, 63-85 (1972)

38. Mariappan, S., Sujith, R.I.: Thermoacoustic instability in a solid rocket motor: non-normality and nonlinear instabilities. J. Fluid Mech. 653, 1-33 (2010)

39. Fotia, M.L., Hoke, J.L., Scientific, I.: Propellant plenum dynamics in a two-dimensional rotating detonation experiment. In: 52nd Aerospace Sciences Meeting, pp. 1-10 (2014)

40. Schwer, D., Kailasanath, K.: Feedback into mixture plenums in rotating detonation engines. In: 50th AIAA Aerospace Sciences Meeting including the New Horizons Forum and Aerospace Exposition, Nashville, TN (2012)

41. Gruber, S., Skews, B.: Weak shock wave reflection from concave surfaces. Exp. Fluids. 54, 1571 (2013)

42. Bedick, C., Sisler, A., Ferguson, D., Strakey, P., Nix, A., Billips, D.: Development of a lab-scale experimental testing platform for rotating detonation engine inlets. In: 55th AIAA Aerospace Sciences Meeting, Grapevine, TX (2017)

43. Ciccarelli, G., Dorofeev, S.: Flame acceleration and transition to detonation in ducts. Prog. Energy Combust. Sci. 34, 499-550 (2008)

44. Nakayama, H., Moriya, T., Kasahara, J., Matsuo, A., Sasamoto, Y., Funaki, I.: Stable detonation wave propagation in rectangular-cross-section curved channels. Combust. Flame. 159, 859-869 (2012)

45. Pan, Z., Fan, B., Zhang, X., Gui, M., Dong, G.: Wavelet pattern and self-sustained mechanism of gaseous detonation rotating in a coaxial cylinder. Combust. Flame. 158, 2220-2228 (2011)

46. Lee, J.H.S.: The Detonation Phenomenon. Cambridge University Press (2008) 